\title{
Reaction-diffusion problems under non-local boundary conditions with blow-up solutions
}

\author{
Monica Marras ${ }^{1}$ and Stella Vernier Piro ${ }^{2^{*}}$
}

"Correspondence: svernier@unica.it 2Dipartimento di Matematica e Informatica, Universita' di Cagliari, via Ospedale 72, Cagliari, Italy Full list of author information is available at the end of the article

\begin{abstract}
This paper deals with blow-up solutions to a class of reaction-diffusion equations under non-local boundary conditions. We prove that under certain conditions on the data the blow-up will occur at some finite time and when the blow-up does occur, lower and upper bounds are derived.
\end{abstract}

MSC: $35 \mathrm{~K} 55 ; 35 \mathrm{~K} 60$

Keywords: blow-up; nonlinear parabolic equations

\section{Introduction}

Quittner and Souplet in [1] consider different classes of reaction-diffusion problems with non-local source terms involving space integrals and investigate under what conditions the solutions blow up or exist globally (see also [2, 3]). Recently Song [4] has considered parabolic problems under Dirichlet or Neumann boundary conditions, containing a nonlocal term in the nonlinearities and, for solutions that blow up at some finite time, they derive lower bounds for the blow-up time. For other contributions in this field, see [3, 5, 6] and [7-9] for reaction-diffusion equations, and see [2] and [10-12] for systems.

In this paper we consider a class of reaction-diffusion equations where a space integral is present on the boundary condition and time dependent coefficients are present both in the nonlinearity term and in the boundary condition. Our aim is to introduce conditions on the data and geometry of the spatial domain, sufficient for the solution to blow up in finite time $t^{*}$. Moreover, lower and upper bounds are derived. More precisely we consider the following problem:

$$
\begin{aligned}
& u_{t}=\Delta u+K_{1}(t) f(u), \quad x \in \Omega, t \in\left(0, t^{*}\right), \\
& \frac{\partial u}{\partial v}=K_{2}(t) \int_{\Omega} g(u) d x, \quad x \in \partial \Omega, t \in\left(0, t^{*}\right), \\
& u(x, 0)=u_{0}(x) \geq 0, \quad x \in \Omega,
\end{aligned}
$$

where $\Omega$ is a bounded domain in $\mathbb{R}^{N}$, with smooth boundary, $\frac{\partial u}{\partial v}$ is the outward normal derivative of $u$ on the boundary $\partial \Omega, f(s), g(s), u_{0}(x)$ are smooth non-negative functions, $t^{*}$ is the blow-up time if blow-up occurs, and the time dependent coefficients $K_{i}(t), i=1,2$, are positive and regular functions. Moreover, $u_{0}(x)$ satisfies the compatibility condition on the boundary. Note that $u \geq 0$ for the maximum principle. The results are based on some Sobolev type inequalities [12] and differential inequality techniques.

○2014 Marras and Vernier Piro; licensee Springer. This is an Open Access article distributed under the terms of the Creative Commons Attribution License (http://creativecommons.org/licenses/by/2.0), which permits unrestricted use, distribution, and reproduction in any medium, provided the original work is properly cited. 
If $K_{2}=0$, (2) becomes the usual Neumann boundary condition and we obtain the PaynePhilippin's result contained in [5], Theorem 2.

Now we state the main theorems of this paper.

Theorem 1.1 Let $u(x, t)$ be a (non-negative) classical solution of problem (1)-(3) with $\Omega$ a bounded convex domain in $\mathbb{R}^{3}$ with the origin inside.

Assume that the functions $f$ and $g$ satisfy

$$
0 \leq f(s) \leq s^{p}, \quad s>0, \quad 0 \leq g(\eta) \leq \eta^{q}, \quad \eta>0, p>q \geq 2,
$$

where

$$
n>\max \left(\frac{1}{p-1}, \frac{q}{2(p-1)}\right)
$$

and

$$
\frac{K_{1}^{\prime}(t)}{K_{1}(t)} \leq \beta, \quad \beta \geq 0
$$

Define

$$
\Phi(t):=K_{1}^{2 n}(t) \int_{\Omega} u^{2 n(p-1)} d x .
$$

If the solution $u(x, t)$ becomes unbounded in $\Phi$-measure at time $t^{*}$, then

$$
t^{*} \geq T
$$

where $T$ is implicitly given as

$$
\int_{0}^{T} \tilde{B}(\tau) e^{2 \mathcal{A}(\tau)} d \tau=\frac{1}{2}(\Phi(0))^{-2},
$$

where $\mathcal{A}(t)$ and $\tilde{B}(t)$ are two suitable positive functions.

Theorem 1.2 Let $u(x, t)$ be a (non-negative) classical solution of (1)-(3) with $\Omega$ a bounded domain in $\mathbb{R}^{N}$. Assume that the functions $f$ and $g$ satisfy

$$
f(s) \geq s^{p}, \quad s>0, \quad g(\eta) \geq \eta^{q}, \quad \eta>0, p>q>1 .
$$

Moreover, assume

$$
\lim _{t \rightarrow \infty} \int_{0}^{t} K(\tau) d \tau=\infty, \quad K(t)=|\partial \Omega||\Omega|^{1-q} K_{2}(t)+|\Omega|^{1-p}(\Psi(0))^{p-q} K_{1}(t),
$$

with

$$
\Psi(t)=\int_{\Omega} u(x, t) d x .
$$

Then no solution $u(x, t)$ can exist for all time, but it blows up in $L^{1}$ and hence in $L^{r}$ norm, $r>1$, at time $t^{*}$, with $t^{*} \leq T$ and $T$ implicitly defined by

$$
\int_{0}^{T} K(\tau) d \tau=\frac{(\Psi(0))^{1-q}}{q-1} .
$$


The paper is organized as follows. In Section 2 we obtain a lower bound for $t^{*}$ under the hypothesis of convexity of $\Omega$ and suitable conditions on data and time dependent coefficients.

In Section 3, we consider the problem (1)-(3) under conditions on the data which ensure that no solution can exist for all time. In fact the solution blows up at some finite time $t^{*}$ in $L^{1}$ and hence in $L^{r}$ norm $(r>1)$ and upper bounds for $t^{*}$ are derived. We note that we obtain blow-up, even if the coefficients are constants and also for $K_{i}(t)$ functions decreasing not too fast at infinity.

For physical motivation of such problems we refer the reader to [13-16] and the references therein.

\section{Lower bounds}

First we state an inequality that plays a basic role in the proofs of our results.

Lemma 2.1 Let $W$ be any non-negative $C^{1}$ function and $\Omega$ a bounded convex domain in $\mathbb{R}^{N}, N \geq 2$, with the origin inside. Then, for any $m \geq 1$, the following inequality holds:

$$
\begin{aligned}
\int_{\partial \Omega} W^{m} d s \leq & |\Omega|^{\frac{1}{m+1}}\left\{\frac{N}{\rho_{0}}\left[\int_{\Omega} W^{m+1} d x\right]^{\frac{m}{m+1}}\right. \\
& \left.+\frac{m d}{\rho_{0}}\left[\int_{\Omega} W^{m+1} d x\right]^{\frac{m-1}{2(m+1)}}\left[\int_{\Omega} W^{m-1}|\nabla W|^{2} d x\right]^{\frac{1}{2}}\right\},
\end{aligned}
$$

where

$$
\rho_{0}:=\inf _{\partial \Omega}\left(\sum_{1}^{N} x_{i} v_{i}\right)>0 \quad \text { and } \quad d^{2}:=\max _{\bar{\Omega}}\left(\sum_{1}^{N} x_{i} x_{i}\right), \quad i=1, \ldots, N .
$$

Proof We start from the following Sobolev type inequality derived in [8] and [9]:

$$
\int_{\partial \Omega} W^{m} d s \leq \frac{N}{\rho_{0}} \int_{\Omega} W^{m} d x+\frac{m d}{\rho_{0}} \int_{\Omega} W^{m-1}|\nabla W| d x .
$$

By applying the Hölder inequality, the first term on the right of (16) becomes

$$
\int_{\Omega} W^{m} d x \leq|\Omega|^{\frac{1}{m+1}}\left(\int_{\Omega} W^{m+1} d x\right)^{\frac{m}{m+1}}
$$

Moreover, by applying the Schwarz and Hölder inequalities to the second term on the right of (16) we get

$$
\begin{aligned}
\int_{\Omega} W^{m-1}|\nabla W| d x & \leq\left(\int_{\Omega} W^{m-1} d x\right)^{\frac{1}{2}}\left(\int_{\Omega} W^{m-1}|\nabla W|^{2} d x\right)^{\frac{1}{2}} \\
& \leq|\Omega|^{\frac{1}{m+1}}\left(\int_{\Omega} W^{m+1} d x\right)^{\frac{m-1}{2(m+1)}}\left(\int_{\Omega} W^{m-1}|\nabla W|^{2} d x\right)^{\frac{1}{2}} .
\end{aligned}
$$

By inserting (17) and (18) in (16), we get (14). 
Our aim is now to derive a lower bound for $t^{*}$.

We assume that $u(x, t)$ becomes unbounded at some finite time $t^{*}$, and under the conditions of Theorem 1.1, we derive a lower bound for $t^{*}$, which works for values of $u_{0}$ not too small.

For brevity, we let $z=2 n(p-1)$ and $K_{i}:=K_{i}(t), i=1,2$. We recall that from (5) we have $z>2$. We compute

$$
\Phi^{\prime}(t) \leq 2 n \frac{K_{1}^{\prime}}{K_{1}} \Phi+z K_{1}^{2 n} \int_{\Omega} u^{z-1}\left[\Delta u+K_{1} f(u)\right] d x .
$$

By applying the divergence theorem, boundary conditions (2) and (4), we have

$$
\begin{aligned}
\int_{\Omega} u^{z-1} \Delta u & =\int_{\partial \Omega} u^{z-1} u_{\nu} d s-[z-1] \int_{\Omega} u^{z-2}|\nabla u|^{2} d x \\
& \leq K_{2}(t) \int_{\Omega} u^{q} d x \int_{\partial \Omega} u^{z-1} d s-[z-1] \int_{\Omega} u^{z-2}|\nabla u|^{2} d x .
\end{aligned}
$$

For convenience, set $v=u^{\frac{z}{2}}$; we get

$$
|\nabla v|^{2}=\frac{z^{2}}{4} u^{z-2}|\nabla u|^{2}
$$

We now replace (20), (21) in (19) and use (6) to obtain

$$
\begin{aligned}
\Phi^{\prime}(t) \leq & 2 n \beta \Phi+z K_{1}^{2 n+1} \int_{\Omega} v^{2+\frac{1}{n}} d x-\frac{4(z-1)}{z} K_{1}^{2 n} \int_{\Omega}|\nabla v|^{2} d x \\
& +z K_{1}^{2 n} K_{2} \int_{\Omega} u^{q} d x \int_{\partial \Omega} u^{z-1} d s .
\end{aligned}
$$

To estimate the second term on the right-hand side of (22) we use the inequality (3.8) in [5], i.e.

$$
\begin{aligned}
K_{1}^{2 n+1} \int_{\Omega} v^{2+\frac{1}{n}} d x & \leq \frac{n-1}{n \gamma} K_{1}^{2 n} \int_{\Omega} v^{2} d x+\frac{\gamma^{n-1}}{n} K_{1}^{3 n} \int_{\Omega} v^{3} d x \\
& =\frac{n-1}{n \gamma} \Phi+\frac{\gamma^{n-1}}{n} K_{1}^{3 n} \int_{\Omega} v^{3} d x,
\end{aligned}
$$

where $\gamma$ is an arbitrary positive constant to be chosen later.

Now we estimate the second term in (23) by using (7) and the inequalities (48), (51) in [5], valid in a convex domain $\Omega \subset \mathbb{R}^{3}$. We obtain

$$
\begin{aligned}
K_{1}^{3 n} \int_{\Omega} v^{3} d x & \leq\left[\lambda \Phi+\mu\left(K_{1}^{2 n} \Phi \int_{\Omega}|\nabla v|^{2} d x\right)^{\frac{1}{2}}\right]^{\frac{3}{2}} \\
& \leq \sqrt{2}\left[\lambda^{\frac{3}{2}} \Phi^{\frac{3}{2}}+\mu^{\frac{3}{2}} \Phi^{\frac{3}{4}}\left(K_{1}^{2 n} \int_{\Omega}|\nabla v|^{2} d x\right)^{\frac{3}{4}}\right] \\
& \leq \sqrt{2}\left[\lambda^{\frac{3}{2}} \Phi^{\frac{3}{2}}+\mu^{\frac{3}{2}}\left(\frac{1}{4 \sigma^{3}} \Phi^{3}+\frac{3}{4} \sigma K_{1}^{2 n} \int_{\Omega}|\nabla v|^{2} d x\right)\right],
\end{aligned}
$$


with $\lambda=\frac{\sqrt{3}}{2 \rho_{0}}, \mu=\frac{1}{\sqrt{3}}\left(1+\frac{d}{\rho_{0}}\right), \rho_{0}$ and $d$ in (15) with $N=3$, and $\sigma>0$ is an arbitrary constant.

Note that deriving the second and third inequality in (24), we make use of the inequality

$$
(a+b)^{\frac{3}{2}} \leq \sqrt{2}\left(a^{\frac{3}{2}}+b^{\frac{3}{2}}\right), \quad a>0, b>0,
$$

and of the arithmetic inequality

$$
a^{r} b^{1-r} \leq r a+(1-r) b, \quad a>0, b>0, r \in(0,1) .
$$

Inserting (23) and (24) in (22) we get

$$
\begin{aligned}
\Phi^{\prime}(t) \leq & \left(2 n \beta+\frac{2(n-1)(p-1)}{\gamma}\right) \Phi \\
& +2^{\frac{3}{2}} \gamma^{n-1}(p-1) \lambda^{\frac{3}{2}} \Phi^{\frac{3}{2}}+\frac{\sqrt{2}}{2} \frac{(p-1) \gamma^{n-1} \mu^{\frac{3}{2}}}{\sigma^{3}} \Phi^{3} \\
& +\left\{\frac{3 \sqrt{2}}{2}(p-1) \gamma^{n-1} \mu^{\frac{3}{2}} \sigma-\frac{4[z-1]}{z}\right\} J+z K_{1}^{2 n} K_{2} \int_{\Omega} u^{q} d x \int_{\partial \Omega} u^{z-1} d s,
\end{aligned}
$$

where $J:=K_{1}^{2 n} \int_{\Omega}|\nabla v|^{2} d x$.

We now estimate separately the two factors in the last term in (27). For the first, making use of Lemma 2.1 with $m=z-1$ and $W=u, N=3$ we have

$$
\begin{aligned}
& K_{1}^{2 n} \int_{\partial \Omega} u^{z-1} d s \\
& \quad \leq K_{1}^{2 n}|\Omega|^{\frac{1}{z}}\left\{\frac{3}{\rho_{0}}\left(\int_{\Omega} u^{z} d x\right)^{\frac{z-1}{z}}+(z-1) \frac{d}{\rho_{0}}\left(\int_{\Omega} u^{z} d x\right)^{\frac{z-2}{2 z}}\left(\int_{\Omega} u^{z-2}|\nabla u|^{2} d x\right)^{\frac{1}{2}}\right\} \\
& \quad \leq|\Omega|^{\frac{1}{z}}\left\{K_{1}^{\frac{1}{p-1}} \frac{3}{\rho_{0}} \Phi^{\frac{z-1}{z}}+2 \frac{(z-1)}{z} \frac{d}{\rho_{0}} K_{1}^{\frac{1}{p-1}} \Phi^{\frac{z-2}{2 z}} J^{\frac{1}{2}}\right\} .
\end{aligned}
$$

In the second, by using the Hölder inequality and hypothesis (5), we obtain

$$
\int_{\Omega} u^{q} d x \leq\left(\int_{\Omega} u^{z} d x\right)^{\frac{q}{z}}|\Omega|^{\frac{z-q}{z}}=\Phi^{\frac{q}{z}}|\Omega|^{1-\frac{q}{z}} K_{1}^{-\frac{q}{p-1}} .
$$

By using (28) and (29), we get

$$
\begin{aligned}
& K_{1}^{2 n} \int_{\partial \Omega} u^{z-1} d s \int_{\Omega} u^{q} d x \\
& \quad \leq|\Omega|^{\frac{1}{z}}\left[\frac{3}{\rho_{0}} K_{1}^{\frac{1}{(p-1)}} \Phi^{1-\frac{1}{z}}+\frac{2(z-1)}{z} \frac{d}{\rho_{0}} K_{1}^{\frac{1}{(p-1)}} \Phi^{\frac{z-2}{2 z}} J^{\frac{1}{2}}\right]\left[\Phi^{\frac{q}{z}}|\Omega|^{\frac{z-q}{z}} K_{1}^{-\frac{q}{p-1}}\right] \\
& \quad=|\Omega|^{1+\frac{1-q}{z}} K_{1}^{\frac{1-q}{p-1}}\left[\frac{3}{\rho_{0}} \Phi^{1+\frac{q-1}{z}}+\frac{2(z-1)}{z} \frac{d}{\rho_{0}} \Phi^{\frac{1}{2}+\frac{q-1}{z}} J^{\frac{1}{2}}\right] \\
& \quad \leq|\Omega|^{1+\frac{1-q}{z}} K_{1}^{\frac{1-q}{p-1}}\left[\frac{3}{\rho_{0}} \Phi^{1+\frac{q-1}{z}}+\frac{2(z-1)}{z} \frac{d}{\rho_{0}}\left(\frac{1}{2 \epsilon} \Phi^{1+2 \frac{q-1}{z}}+\frac{\epsilon}{2} J\right)\right],
\end{aligned}
$$

where in the last inequality we use (26) with $\epsilon>0$ arbitrary. 
We now replace (30) in (27) to have

$$
\Phi^{\prime}(t) \leq A \Phi+B \Phi^{\frac{3}{2}}+C \Phi^{3}+D \Phi^{1+\frac{q-1}{z}}+E \Phi^{1+2 \frac{q-1}{z}}+F J
$$

with

$$
\left\{\begin{aligned}
A & :=2 n \beta+\frac{2(n-1)(p-1)}{\gamma}, \\
B & :=(2 \lambda)^{\frac{3}{2}}(p-1) \gamma^{n-1}, \\
C & :=\frac{\sqrt{2}}{2} \frac{(p-1)}{\sigma^{3}} \gamma^{n-1} \mu^{\frac{3}{2}}, \\
D(t): & :=\frac{3}{\rho_{0}} z K_{2} K_{1}^{\frac{1-q}{p-1}}|\Omega|^{1+\frac{1-q}{z}}, \\
E(t):= & \frac{d}{\rho_{0}} \frac{z-1}{\epsilon} K_{2} K_{1}^{\frac{1-q}{p-1}}|\Omega|^{1+\frac{1-q}{z}}, \\
F(t):= & \frac{3}{\sqrt{2}}(p-1) \gamma^{n-1} \mu^{\frac{3}{2}} \sigma-4\left(\frac{z-1}{z}\right) \\
& \quad(z-1) \frac{d}{\rho_{0}} \epsilon K_{2} K_{1}^{\frac{1-q}{p-1}}|\Omega|^{1+\frac{1-q}{z}} .
\end{aligned}\right.
$$

We now choose $\gamma, \sigma, \epsilon$ positive constants such that $F \equiv 0$.

A possible choice of $\gamma, \sigma, \epsilon$, is

$$
\left\{\begin{array}{l}
\gamma:=1, \\
\sigma:=\frac{2 \sqrt{2}}{3} \frac{z-1}{z(p-1) \mu^{\frac{3}{2}}}, \\
\epsilon:=\frac{2 \rho_{0}}{z d} K_{2}^{-1} K_{1}^{\frac{q-1}{p-1}}|\Omega|^{\frac{q-1}{z}-1} .
\end{array}\right.
$$

Then

$$
\left\{\begin{array}{l}
A:=2 n \beta+2(n-1)(p-1), \\
B:=(2 \lambda)^{\frac{3}{2}}(p-1), \\
C:=\frac{(p-1)^{4}}{2^{5}}\left(\frac{3 z}{z-1} \mu^{2}\right)^{3}, \\
D:=\frac{3}{\rho_{0}} z K_{2} K_{1}^{\frac{1-q}{p-1}}|\Omega|^{1+\frac{1-q}{z}}, \\
E:=\frac{z}{2}(z-1)\left(\frac{d}{\rho_{0}} K_{2} K_{1}^{\frac{1-q}{p-1}}|\Omega|^{1+\frac{1-q}{z}}\right)^{2} .
\end{array}\right.
$$

Note that $A, B, C$ are positive constants, whereas $D$ and $E$ may depend on the time through the coefficients $K_{1}$ and $K_{2}$.

In order to simplify (31), set

$$
Q:=\frac{q-1}{2(p-1)}
$$

and we obtain $\Phi^{1+\frac{(q-1)}{z}}=\Phi^{1+\frac{Q}{n}}$ and $\Phi^{1+\frac{2(q-1)}{z}}=\Phi^{1+\frac{2 Q}{n}}$. Now (31) can be rewritten (with $F=0)$ as

$$
\Phi^{\prime}(t) \leq A \Phi+B \Phi^{\frac{3}{2}}+C \Phi^{3}+D(t) \Phi^{1+\frac{Q}{n}}+E(t) \Phi^{1+\frac{2 Q}{n}} .
$$

In the second term in (35) we now use the Schwarz inequality to obtain

$$
\Phi^{\frac{3}{2}}=\Phi^{\frac{3}{4}}\left(\Phi^{3}\right)^{\frac{1}{4}}=\left(C_{1}^{-\frac{1}{3}} \Phi\right)^{\frac{3}{4}}\left(C_{1} \Phi^{3}\right)^{\frac{1}{4}} \leq \frac{3}{4} C_{1}^{-\frac{1}{3}} \Phi+\frac{C_{1}}{4} \Phi^{3} .
$$


Moreover, since $n>Q$, we write

$$
\begin{aligned}
\Phi^{1+\frac{Q}{n}} & =\Phi^{1-\frac{Q}{2 n}}\left(\Phi^{3}\right)^{\frac{Q}{2 n}}=\left(C_{2}^{M_{1}} \Phi\right)^{1-\frac{Q}{2 n}}\left(C_{2} \Phi^{3}\right)^{\frac{Q}{2 n}} \\
& \leq\left(1-\frac{Q}{2 n}\right) C_{2}^{M_{1}} \Phi+\frac{Q}{2 n} C_{2} \Phi^{3}
\end{aligned}
$$

with $M_{1}:=\left(1-\frac{2 n}{Q}\right)^{-1}$, and $C_{2}$ an arbitrary positive constant.

Analogously

$$
\begin{aligned}
\Phi^{1+\frac{2 Q}{n}} & =\Phi^{1-\frac{Q}{n}}\left(\Phi^{3}\right)^{\frac{Q}{n}} \\
& =\left(C_{3}^{M_{2}} \Phi\right)^{1-\frac{Q}{n}}\left(C_{3} \Phi^{3}\right)^{\frac{Q}{n}} \leq\left(1-\frac{Q}{n}\right) C_{3}^{M_{2}} \Phi+\frac{Q}{n} C_{3} \Phi^{3},
\end{aligned}
$$

where we again use (26) with $M_{2}:=\left(1-\frac{n}{Q}\right)^{-1}$, and $C_{3}>0$ an arbitrary positive constant.

Moreover, we insert (36), (37), and (38) in (35) so that the differential inequality (35) can be rewritten as

$$
\Phi^{\prime}(t) \leq \tilde{A} \Phi+\tilde{B} \Phi^{3}
$$

with

$$
\left\{\begin{array}{l}
\tilde{A}(t):=A+E\left(1-\frac{Q}{n}\right) C_{3}^{M_{2}}+D\left(1-\frac{Q}{2 n}\right) C_{2}^{M_{1}}+B \frac{3}{4 C_{1}^{\frac{1}{3}}}, \\
\tilde{B}(t):=C+\frac{Q}{n} E C_{3}+\frac{Q}{2 n} D C_{2}+\frac{C_{1}}{4} B .
\end{array}\right.
$$

From (39) we can write

$$
-\frac{1}{2}\left(\Phi^{-2}\right)^{\prime}=\Phi^{\prime} \Phi^{-3} \leq \tilde{A} \Phi^{-2}+\tilde{B}
$$

and we set $\phi(t):=\Phi^{-2}$ to obtain

$$
\phi^{\prime}+2 \tilde{A} \phi \geq-2 \tilde{B}
$$

Then define $\mathcal{A}(t):=\int_{0}^{t} \tilde{A}(\tau) d \tau$, and (41) may be rewritten as

$$
\left(\phi e^{2 \mathcal{A}(t)}\right)^{\prime} \geq-2 \tilde{B}(t) e^{2 \mathcal{A}(t)} .
$$

From this we obtain

$$
\phi(t) e^{2 \mathcal{A}(t)}-\phi(0) \geq-2 \int_{0}^{t} \tilde{B}(\tau) e^{2 \mathcal{A}(\tau)} d \tau
$$

i.e.

$$
\Phi^{-2}(t)=\phi(t) \geq\left[\phi(0)-2 \int_{0}^{t} \tilde{B}(\tau) e^{2 \mathcal{A}(\tau)} d \tau\right] e^{-2 \mathcal{A}(t)}:=\theta(t) .
$$


Then if $\theta(T)=0$, we have

$$
t^{*} \geq T
$$

Clearly $T$ is implicitly given by (9).

Remark 1 Note that the bound (9) is a good estimate of $t^{*}$ because we consider our problem (1)-(3) with initial data $u_{0}(x)$ not too small. For instance we can choose $u_{0}(x)$ such that $\int_{\Omega} u_{0}^{2 n(p-1)}(x) d x>\frac{\xi}{K_{1}^{2 n}(0)}$.

Remark 2 If $K_{1}$ and $K_{2}$ are constants then $\tilde{A}$ and $\tilde{B}$ are constants.

In this case we have

$$
T=\frac{1}{2 \tilde{A}} \log \left(1+\frac{\tilde{A}}{\tilde{B}}(\Phi(0))^{-2}\right) .
$$

\section{Blow-up of $u$ in finite time and upper bounds. Proof of Theorem 1.2}

In this section we establish that under the hypotheses of Theorem 1.2, no solution can exist for all time, but it blows up in $L^{1}$ and hence in $L^{r}$ norm, $r>1$, at time $t^{*}$. Then an upper bound of $t^{*}$ is obtained.

To this end we compute

$$
\begin{aligned}
\Psi^{\prime}(t) & =\int_{\Omega} \Delta u d x+K_{1}(t) \int_{\Omega} f(u) d x \\
& =K_{2}|\partial \Omega| \int_{\Omega} g(u) d x+K_{1} \int_{\Omega} f(u) d x \geq K_{2}|\partial \Omega| \int_{\Omega} u^{q} d x+K_{1} \int_{\Omega} u^{p} d x,
\end{aligned}
$$

where in the last inequality we used (10).

For any $\delta>1$, by the Hölder inequality

$$
\int_{\Omega} u d x \leq\left(\int_{\Omega} u^{\delta} d x\right)^{\frac{1}{\delta}}|\Omega|^{\frac{\delta-1}{\delta}}
$$

we have

$$
\int_{\Omega} u^{\delta} d x \geq \Psi^{\delta}|\Omega|^{1-\delta} .
$$

By using (44) in (43) we get

$$
\Psi^{\prime}(t) \geq K_{2}(t)|\partial \Omega||\Omega|^{1-q} \Psi^{q}+K_{1}(t)|\Omega|^{1-p} \Psi^{p} .
$$

Now we observe that the function $\Psi$ is non-decreasing, then $\Psi(t) \geq \Psi(0)>0$.

Since $p>q>1$,

$$
\left(\frac{\Psi(t)}{\Psi(0)}\right)^{q} \leq\left(\frac{\Psi(t)}{\Psi(0)}\right)^{p}
$$

i.e.

$$
\Psi^{p}(t) \geq \Psi^{q}(t)(\Psi(0))^{p-q} .
$$


By inserting (46) in (45), we have

$$
\Psi^{\prime}(t) \geq K(t) \Psi^{q}
$$

with $K(t)$ defined in (11).

Now we integrate (47) from 0 to $t$ and we obtain the inequality

$$
(\Psi(t))^{1-q}-(\Psi(0))^{p-q} \leq-(q-1) \int_{0}^{t} K(\tau) d \tau, \quad t \in\left(0, t^{*}\right) .
$$

Using (11) we see that the inequality (48) cannot hold for all time, but $u$ will blow up in $L^{1}$ norm (hence in $L^{r}$ norm, $r>1$ ) at a finite time $t^{*}$ and

$$
\int_{0}^{t^{*}} K(\tau) d \tau \leq \int_{0}^{T} K(\tau) d \tau:=\frac{(\Psi(0))^{1-q}}{q-1}
$$

Moreover, let $k(t)=\int_{0}^{t} K(\tau) d \tau$. Since $k$ is increasing, there exists the inverse function $k^{-1}$ and we can write

$$
t^{*} \leq k^{-1}\left(\frac{(\Psi(0))^{1-q}}{q-1}\right)
$$

which is the desired upper bound of $t^{*}$.

Another upper bound for $t^{*}$ can be obtained by means of a new auxiliary function $\chi(t)$ so defined:

$$
\chi(t)=K_{2}^{\frac{1}{q-1}}(t) \int_{\Omega} u d x, \quad q>1,
$$

with $K_{2}(t)$ satisfies

$$
\frac{K_{2}^{\prime}(t)}{K_{2}(t)} \geq \tilde{\beta}>0
$$

Under this condition no (non-negative) solution $u(x, t)$ of problem (1)-(3) can exist for all time, but it blows up in $\chi$ norm at time $t^{*}$, with $t^{*} \leq T$ and $T$ implicitly defined by

$$
\int_{0}^{T} \tilde{K}(\tau) e^{\tilde{\beta} \tau} d \tau=\frac{(\chi(0))^{1-q}}{q-1}
$$

In fact, following the proof of Theorem 1.2, we obtain the differential inequality

$$
\chi^{\prime}(t) \geq \frac{\tilde{\beta}}{q-1} \chi+\tilde{K}(t) \chi^{q}
$$

where $\tilde{K}(t)=|\partial \Omega||\Omega|^{1-q}+(\chi(0))^{p-q} K_{1} K_{2}^{\frac{1-p}{q-1}}|\Omega|^{1-p}$.

Integrating (53) over $[0, t]$, we obtain

$$
e^{\tilde{\beta} t} \chi(t)^{1-q} \leq(\chi(0))^{1-q}-(q-1) \int_{0}^{t} \tilde{K}(\tau) e^{\tilde{\beta} \tau} d \tau .
$$


We see that the inequality (54) cannot hold for all time, but $u$ will blow up in $\chi$ norm at a finite time $t^{*}$. At the end we get the upper bound $T$, with $T$ implicitly defined by (52).

Remark The result can be extended to the case $\tilde{\beta}=0$. In fact, by (53) we get

$$
\chi^{\prime}(t) \geq \tilde{K}(t) \chi^{q}
$$

By assuming that

$$
\lim _{t \rightarrow \infty} \int_{0}^{t} \tilde{K}(\tau) d \tau=\infty,
$$

we conclude that the solution blows up in $\chi$ norm at time $t^{*}$, with $t^{*} \leq T$ and $T$ implicitly defined by

$$
\int_{0}^{T} \tilde{K}(\tau) d \tau=\frac{(\chi(0))^{1-q}}{q-1}
$$

\section{Competing interests}

The authors declare that they have no competing interests.

\section{Authors' contributions}

The authors contributed equally to the writing of this paper. They also read and approved the final manuscript.

\section{Author details}

'Dipartimento di Matematica e Informatica, Universita' di Cagliari, viale Merello 92, Cagliari, Italy. ${ }^{2}$ Dipartimento di Matematica e Informatica, Universita' di Cagliari, via Ospedale 72, Cagliari, Italy.

\section{Authors' information}

The authors are members of the Gruppo Nazionale per l'Analisi Matematica, la Probabilità e le loro Applicazioni (GNAMPA) of the Istituto Nazionale di Alta Matematica (INdAM).

Received: 28 October 2013 Accepted: 9 April 2014 Published: 06 May 2014

\section{References}

1. Quittner, P, Souplet, P: Superlinear Parabolic Problems. Blow-up, Global Existence and Steady States. Birkhäuser Advanced Texts. Birkhäuser, Basel (2007)

2. Marras, M, Vernier Piro, S: Blow-up phenomena in reaction-diffusion systems. Discrete Contin. Dyn. Syst. 32(11), 4001-4014 (2012)

3. Marras, M, Vernier Piro, S: On global existence and bounds for blow-up time in non-linear parabolic problems with time dependent coefficients. Discrete Contin. Dyn. Syst. 2013, suppl., 535-544 (2013)

4. Song, JC: Lower bounds for the blow-up time in a non-local reaction-diffusion problem. Appl. Math. Lett. 24, 793-796 (2011)

5. Payne, LE, Philippin, GA: Blow up phenomena in parabolic problems with time dependent coefficients under Neumann boundary conditions. Proc. R. Soc. Edinb. 142, 625-631 (2012)

6. Payne, LE, Philippin, GA: Blow up in a class of non-linear parabolic problems with time dependent coefficients under Robin type boundary conditions. Appl. Anal. 91, 2245-2256 (2012)

7. Payne, LE, Philippin, GA, Vernier Piro, S: Blow up phenomena for a semilinear heat equation with nonlinear boundary condition, I. Z. Angew. Math. Phys. 61, 999-1007 (2010)

8. Payne, LE, Philippin, GA, Vernier Piro, S: Blow up phenomena for a semilinear heat equation with nonlinear boundary condition, II. Nonlinear Anal. 73, 971-978 (2010)

9. Payne, LE, Schaefer, PW: Lower bounds for blow-up time in parabolic problems under Neumann boundary conditions. Appl. Anal. 85, 1301-1311 (2006)

10. Marras, M: Bounds for blow-up time in nonlinear parabolic systems under various boundary conditions. Numer Funct. Anal. Optim. 32, 453-468 (2011)

11. Marras, M, Vernier Piro, S: Bounds for blow-up time in nonlinear parabolic system. Discrete Contin. Dyn. Syst. 2011, suppl., 1025-1031 (2011)

12. Payne, LE, Schaefer, PW: Blow-up phenomena for some nonlinear parabolic systems. Int. J. Pure Appl. Math. 42, 193-202 (2008)

13. Hu, B, Yin, HM: Semilinear parabolic equations with prescribed energy. Rend. Circ. Mat. Palermo 44, $479-505$ (1995)

14. Rubinstein, J, Sternberg, P: Nonlocal reaction-diffusion equations and nucleation. IMA J. Appl. Math. 48, 249-264 (1992) 
15. Marras, M, Vernier Piro, S: Blow up and decay bounds in quasilinear parabolic problems. Discrete Contin. Dyn. Syst. 2007, suppl., 704-712 (2007)

16. Payne, LE, Philippin, GA: Blow up phenomena for a class of parabolic systems with time dependent coefficients. Appl. Math. 3, 325-330 (2012)

10.1186/1029-242X-2014-167

Cite this article as: Marras and Vernier Piro: Reaction-diffusion problems under non-local boundary conditions with blow-up solutions. Journal of Inequalities and Applications 2014, 2014:167

Submit your manuscript to a SpringerOpen ${ }^{\circ}$ journal and benefit from:

- Convenient online submission

- Rigorous peer review

- Immediate publication on acceptance

Open access: articles freely available online

- High visibility within the field

- Retaining the copyright to your article

Submit your next manuscript at $\boldsymbol{s p r i n g e r o p e n . c o m ~}$ 